\title{
Self-organizing Home Networking based on Cognitive Radio Technologies
}

\author{
Z. Wang ${ }^{\dagger}$, J. Ansari ${ }^{\star}$, V. Atanasovski ${ }^{\dagger \dagger}$, D. Denkovski ${ }^{\dagger \dagger}$, T. Farnham ${ }^{\diamond}$, L. Gavrilovska ${ }^{\dagger \dagger}$, A. Gefflaut ${ }^{\dagger}$, \\ R. Manfrin ${ }^{\star \star}$, E. Meshkova ${ }^{\star}$, J. Nasreddine ${ }^{\star}$, K. Rerkrai ${ }^{\star}$, M. Sooriyabandara ${ }^{\diamond}$, A. Zanella ${ }^{\star \star}$ \\ * Institute for Networked Systems, RWTH Aachen University, Germany \\ $\dagger$ European Microsoft Innovation Centre, Germany, ${ }^{\diamond}$ Toshiba European Research Laboratory, UK \\ $\dagger^{\dagger}$ Faculty of Electrical Engineering, Ss. Cyril and Mehtodius, Skopje, Macedonia

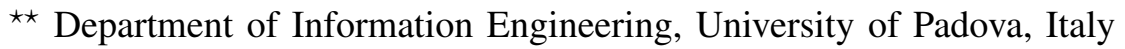 \\ email: jad@inets.rwth-aachen.de
}

\begin{abstract}
The increasing complexity of the future wireless networks leads to the requirement for self-organization. This is true especially in home networking where users are typically not networking professionals and cannot be expected to perform complex optimization and management tasks. In this context, cognitive radio concept combining cross-layer optimization and learning mechanisms is a promising solution. We demonstrate a cognitive home networking prototype, which addresses practical problems users face with the present-day wireless networks at home. The prototype shows how nodes using IEEE 802.11 radios and WARP boards operate under the Cognitive Resource Manager (CRM). The nodes achieve the desired performance by handling network dynamics and controlling parameters taking independent or cooperative decisions and operating in different layers of the protocol stack. This is done using multiple control loops which are supported by the CRM architecture. We demonstrate the use of machine learning for online estimation of network activity patterns to enable more efficient Dynamic Spectrum Access (DSA) using Hidden Semi-Markov Models (HSMM). The demonstration showcases dynamic spectrum allocation and policy-based behavioral changes in a home environment, where several multimedia streams and data communication flows are competing against each other and against external, also primary, interferers.
\end{abstract}

\section{INTRODUCTION}

The huge increase in user demand for wireless communication and respective application performance requirements made wireless environment very complex and difficult to manage by humans. Hence, Cognitive Radio (CR) concept is becoming a need for future wireless networks since it enables the deployment of self-organizing networks that require the minimum of human intervention. However, most of the cognitive radio prototyping have been focusing on either dynamic spectrum access in broadcasting networks such as TV or military and emergency scenarios.

A cooperation project, ARAGORN, between academia and industry has been developing CR technologies that can be used immediately to enhance ISM-band operations, and have also long-term impact in DSA context [1]. A first demonstration of the home networking prototype developed in ARAGORN was presented in DySPAN 2010 [2]. To our best knowledge, this was the first public demonstration of usability of CR networks in such context. In this demonstration we will showcase an extension of the solutions presented in [2], namely by (a) integrating multiple control loops handing optimization processes running on different time-scales across the protocol stack, as well as between different nodes, (b) employing learning mechanisms, (c) application layer control, (d) cooperation, (e) introducing heterogeneously by adding a WARP Software Define Radio (SDR) platform [3] with a DSA-enabled MAC protocol similar to [4].

\section{Demonstration Description ANd Background}

\section{A. Motivation}

Most of the European and American households are using Wi-Fi technologies in their homes. In the near future it is likely that due to a large variety of devices and applications, a home network will have to deal with different kind of traffic ranging from regular low bandwidth data to high bandwidth traffic bursts like high resolution video streaming. In addition, a home network may also be subject to interference from local devices or those located in neighboring home networks. They also might be constrained by other higher priority, e.g. primary, networks. Moreover, users of home networks usually do not have the technical expertise to cope with the complexity related to network configuration, diagnostics and optimization. Therefore, there is a need for developing solutions that allow self-organization for home networking scenario with the minimum intervention of human users. CR technologies seem to be a promising solution in this context.

In this demonstration we will show how $\mathrm{CR}$ techniques can be integrated with different radio transceivers to enhance user experience and network capacity using a typical home network setting and using both common off-the-shelf (COTS) hardware and emerging SDR platforms. The demonstration is divided into four separate parts: (1) Dynamic Spectrum Access (DSA) in presence of both secondary and primary users, (2) Dynamic Adaptation and Coordination (DAC), (3) Machine Learning and (4) Support for Alternative Wireless Technology.

\section{B. Demonstration Overview}

The main objective of the demonstration is to show that CR technologies can be efficiently implemented through the 
CRM architecture in home networks and enable them to adapt to different situations using such methods as DSA, crosslayer optimization, application adaptation, policy-based prioritization, cooperation and learning components. The different parts of the demonstration, which are briefly described in this section, show how the developed solution and modules work together to enhance network performance.

The Dynamic Spectrum Access part is focused on demonstrating how the developed spectrum sensing framework enables dynamic selection of free spectrum/channel for the best user experience. The demonstration includes several different spectrum monitoring devices and developed over-the-air protocol for reporting measurement information. The DSA mechanism is enhanced by a policy-based framework that includes the use of the Prolog-based Policy Reasoner from SRI, and our extended CoRaL based policy language [5].

The Dynamic Adaptation and Coordination part shows dynamic reaction to congestion and interferences and how CRs can reconfigure themselves to utilize free spectrum, avoid primary users, and deal with congestion. It performs dynamic link and flow optimization at different time scales, where negotiation of radio parameters and improved spectrum usage is achieved through the support of spectrum awareness demonstrated previously, as well as more advanced cooperative behavior. One novel aspect of this demonstration is that it shows how dynamic reaction to policy restrictions is not only regulation based, but can also be driven by user and application priorities and preferences.

As one of the network performance optimization process we deployed the mechanism for online estimation and exploitation of network ON-OFF activity patterns. This information is necessary to predict the channel occupancy and efficiently schedule transmissions of Wi-Fi and WARP nodes on different frequency bands, also taking in account their significantly different constraints on reconfiguration timings. This mechanism is a practical example of Machine Learning techniques being intergraded in the CRM architecture.

In addition to use of Atheros IEEE 802.11 chipsets with modified device drivers that allow better access to low-level functionalities [6], we also integrate the WARP board in our CRM framework as the Support for Alternative Wireless Technology.

\section{Demonstration Setup And Components}

The logical architecture of the demonstration includes all the major components that one would find in a realistic home network such as PCs, external primary and secondary interferers. We deploy policy server, several spectrum power sensors, and user devices that include the implementation of a network stack and a Cognitive Resource Manager as the key element supporting the cognitive facilities.

The demonstration setup consists from commercially available hardware components and SDR platforms, which are integrated together through custom made software and interfaces. All components run on ordinary PCs with Windows as operating system, but the network interface functionalities are extended by using virtual interface technology. The CRM and all the dynamic adaptation software are developed by the ARAGORN consortium [1], and can be run on different operating systems [7], [8]. The demonstration is focused on home networking in $2.4 \mathrm{GHz}$ ISM frequency band.

The CRM framework developed in ARAGORN is responsible of controlling and tuning the parameters of the different nodes and protocol layers in order to (a) optimize the use of resources, (b) respect hardware reconfiguration constraints imposed by different radio boards, (c) satisfy the network stakeholders. We show how the CRM reconfigures radios, parameters of different MAC protocols, network layer parameters and applications while satisfying the policies and priorities set by the different stakeholders. In particular the demonstration shows also the possibility of performing utilitybased optimization and application adaptation using the open interface developed by ARAGORN (i.e. Common APplication Requirements Interface or CAPRI). We also make predictions on spectrum usage based on the online structure learning of HSMMs. As part of the demonstration we also display spectrum sensing enabled by a number of SDR platforms, which are CalRadio, WARP, USRP2 (Universal Software Radio Platform) radios, Miniscanner from University of Dortmund, WiSpy equipments that are used as spectrum monitoring devices. We have also a separate PC driven source to generate background interference. As primary users, we use IEEE 802.15.4 radios using narrowband low transmit power to show the sensitivity and agility of our scheme. We monitor the performance of these primary nodes to estimate interference caused by the demonstrated home devices, which are treated as the secondary network in this case.

\section{ACKNOWLEDGMENT}

The authors would like to thank European Union (ARAGORN Project), and the German Research Foundation through UMIC Research Centre for partial financial support. We thank all the collaborators in the ARAGORN consortium for their contributions and lively discussions.

\section{REFERENCES}

[1] ARAGORN project, http://www.ict-aragorn.eu [Last visited 04.03.2011].

[2] V. Atanasovski et. al, "Cognitive radio for home networking," in Demontrated at IEEE DySPAN 2010, Singapore, April 2010.

[3] A. Khattab et. al, "WARP: a flexible platform for clean-slate wireless medium access protocol design," SIGMOBILE Mob. Comput. Commun. Rev., vol. 12, no. 1, pp. 56-58, 2008.

[4] J. Ansari, X. Zhang and P. Mähönen, "A Decentralized MAC for Opportunistic Spectrum Access in Cognitive Wireless Networks," in Proceedings of the ACM SIGMOBILE Workshop on Cognitive Wireless Networking, 2010.

[5] D. Denkovski et. al, "Novel policy reasoning architecture for cognitive radio environments," in Proc. of IEEE Globecom, Miami, USA, Dec. 2010.

[6] R. Chandra et. al, "A case for adapting channel width in wireless networks," in Proc. of ACM SIGCOMM, 2008, pp. 135-146.

[7] T. Cai et. al, "Implementation of cognitive resource management on LTE platform," in Proc. of IEEE PIMRC, Istanbul, Turkey, 2010.

[8] E. Meshkova et. al, "Using Cognitive Radio Principles for Wireless Resource Management in Home Networking," in Prof. of IEEE CCNC. 